\title{
Invariant Region, Endemic Equilibria and Stability Analysis
}

\author{
P. Mafuta*, J. Mushanyu, G. Nhawu \\ Department of Mathematics, University of Zimbabwe Box MP 167, Mount Pleasant, Harare, Zimbabwe
}

\begin{abstract}
In this manuscript, the ideas of Lyapunov, Barbalat, Birkhoff and Rota have been explored on the deterministic model A that describes human population, whose state variables and parameters are assumed to be non-negative. This approach is ideally suitable for S.I models where there is no disease induced death rate, for instance chancroid and trichomoniasis. We have put forward and proved three conjectures, that is, the system of equations that describes model $A$ is dissipative, for an endemic equilibrium point $Q^{*}$ of such a system there exist a strict Lyapunov function and that an endemic equilibrium point $Q^{*}$ is both globally and locally asymptotically stable whenever it exists.
\end{abstract}

Key words: Dissipative; Lyapunov function; Asymptotic Stability

\section{Introduction}

A function $V(x)$ on a region $\Omega$ of state space and containing an equilibrium point $Q^{*}$ is called a Lyapunov function, if it satisfies the following three conditions:

i $V(x)$ is continuous and has continuous first order partial derivatives,

ii $V(x)$ has a unique minimum at $Q^{*}$ with respect to all other points in a region $\Omega$,

iii the function $V^{\prime}(x(t))=\nabla V(x) f(x)$ satisfies $V^{\prime}(x(t)) \leq 0$ for all $x(t) \in \Omega$. [1,2]

The stability analysis of equilibrium points has been dealt with by Lyapunov in his theorem which states that: "If there exist a Lyapunov function for an equilibrium point $Q^{*}$ then $Q^{*}$ is a stable equilibrium point. If further, the function $V^{\prime}(x(t)) \leq 0$ for every point $x(t) \in \Omega$ then the stability is asymptotic." Here we apply Lyapunov's ideas to S.I models where there is no disease induced death. [1,2]

Theorem 1. Let $A$ be a deterministic model that describes human population, $N(t)$ say, whose state variable and parameters are assumed to be non-negative and that there is no disease induced death. Let $S_{i}(t)$ and $I_{i}(t)$ denote the finite number of compartments for susceptible individuals and infected individuals respectively at a given time $t$ for $i=1,2,3, \ldots, n$. Let $\mu$ and $\Lambda$ denote the natural death rate and recruitment rate respectively, where $\mu>0$ and $\Lambda>0$. Then the system of equations that describes a model $A$ is dissipative, that is all the solutions are uniformly bounded on $\Omega \in \mathbb{R}_{+}^{2 n}$.

Proof. Let $\left(S_{1}, I_{1}, S_{2}, I_{2}, S_{3}, I_{3}, \ldots, S_{n}, I_{n}\right) \in \mathbb{R}_{+}^{2 n}$ be any solutions with non-negative initial conditions and set $S(t)=\sum_{i=1}^{n} S_{i}(t), I(t)=\sum_{i=1}^{n} I_{i}(t)$. It follows that $N(t)=S(t)+I(t)$. Using a theorem on differential inequality (Birkhoff and Rota, 1982) [7], it follows that

$\lim _{t \rightarrow \infty} S(t) \leq \frac{\Lambda}{\mu}$

Taking the derivative of $N(t)$ along a solution path of the system gives

$N^{\prime}(t)=S^{\prime}(t)+I^{\prime}(t)$

that is

$$
N^{\prime}(t)=\Lambda-\mu N(t) .
$$

All parameters and state variables of the system are assumed to be non-negative for $t \geq 0$ since $A$ is a model that monitors human population, hence $0 \leq N(t)$. Now by solving the above linear first order differential equation (1) and considering the fact that $\frac{\Lambda e^{-\mu t}}{\mu}>0$, we obtain the following result:

$0<N(t)<\frac{\Lambda}{\mu}$ as $\rightarrow \infty$.

By setting $k=\frac{\Lambda}{\mu}>0$ we conclude that all feasible solutions of the system that describes model $A$ are bounded in $\Omega \in \mathbb{R}_{+}^{2 n}$ where $\Omega=\left\{\left(S_{1}, I_{1}, S_{2}, I_{2}, S_{3}, I_{3}, \ldots, S_{n}, I_{n}\right): 0 \leq N(t) \leq k\right\}$.

The argument above shows that $\Omega$ is positively invariant and it is sufficient to consider solutions in $\Omega$.

Existence, uniqueness and continuation holds in $\Omega$.

Theorem 2. Let A be a deterministic model that satisfies all the conditions of theorem (1) above. Let $Q^{*}$ be an endemic equilibrium point of the system that describes a model A whenever such an equilibrium 
point exist. Then there exists a strict Lyapunov function $V(x)$ for an endemic equilibrium point $Q^{*}$.

Proof. Let $Q^{*}=\left(S_{1}^{*}, I_{1}^{*}, S_{2}^{*}, I_{2}^{*}, S_{3}^{*}, I_{3}^{*}, \ldots, S_{n}^{*}, I_{n}^{*}\right)$ and set $S_{1}=x_{1}, I_{1}=x_{2}, S_{2}=x_{3}, I_{2}=x_{4}, S_{3}=x_{5}, I_{3}=$ $x_{6}, \ldots, S_{n}=x_{2 n-1}$ and $I_{n}=x_{2 n}$.

Define a function $V(x)=\left(x_{1}, x_{2}, x_{3}, x_{4}, x_{5}, x_{6}, \ldots, x_{2 n-1}, x_{2 n}\right)$ by:

$$
V(x)=\sum_{i=1}^{2 n}\left(x_{i}^{*}-x_{i}\right)^{2}
$$

Clearly, $V(x)$ is an entire function, since it is a polynomial, hence $V(x)$ is a continuous function. Moreover,

$$
\frac{\partial V}{\partial x_{i}}=-2\left(x_{i}^{*}-x_{i}\right), \quad i=1,2,3,4, \ldots, 2 n
$$

This implies that the first order partial derivatives of $V(x)$ are continuous for each $x_{i}$. Now by equating the partial derivatives to zero and solve for $x_{i}$ we get:

$$
x_{i}=x_{i}^{*}, \quad i=1,2,3,4, \ldots, 2 n
$$

It follows that the endemic equilibrium point $Q^{*}$ is the only stationary point of the function $V(x)$. In addition,

$$
\frac{\partial^{2} V(x)}{\partial x_{i}^{2}}=2, \quad i=1,2,3,4, \ldots, 2 n
$$

it is clear that the second partial derivative of the function $V(x)$ with respect to $x_{i}$ for $i=1,2,3,4, \ldots, 2 n$, is positive, hence the endemic equilibrium point $Q^{*}$ is a global minimum point of the function $V(x)$, for all $x \in \mathbb{R}_{+}^{2 n}$ and $V^{\prime}=0$ only at the endemic equilibrium point $Q^{*}$.

Therefore to conclude that the function $V(x)$ is a strict Lyapunov function, it suffices to show that,

the rate of change of the function $V(x)$ with respect to time $t$, denoted $V^{\prime}(x(t))$ is less than zero as $t$ approaches infinity. Now

$V^{\prime}(t)=-2 \sum_{i=1}^{2 n}\left(x_{i}^{*}-x_{i}\right) x_{i}^{\prime}(t)$.

Also, since

It implies that

$$
N^{\prime}(t)=\Lambda-\mu N(t)
$$

$$
0 \leq N^{\prime}(t)<\Lambda
$$

Hence, the derivatives are also bounded, that is, $x_{i}^{\prime}(t) \in L^{\infty}$. Thus

$-2 \sum_{i=1}^{2 n}\left(x_{i}^{*}-x_{i}\right) x_{i}^{\prime}(t)<-2 \Lambda \sum_{i=1}^{2 n}\left(x_{i}^{*}-x_{i}\right)$.

Therefore

$V^{\prime}(t)<-2 \Lambda \sum_{i=1}^{2 n}\left(x_{i}^{*}-x_{i}\right)$.

Furthermore, by using the result derived from Barbalat lemma (Barbalat, 1959),

$\sum_{i=1}^{2 n}\left(x_{i}^{*}-x_{i}\right) \rightarrow 0$ as $t \rightarrow \infty$. [1,2]

Hence $V^{\prime}(x(t))<0$, this implies that the function $V(x)$ is a strict Lyapunov function for $Q^{*}$ as desired.

Theorem 3. Let $A$ be a deterministic model with the same conditions as in theorem (1) and $Q^{*}$ be an endemic equilibrum point whenever it exist as in theorem (2). Then the endemic equilibrium point $Q_{-}$is both globally and locally asymptotically stable whenever it exist.

Proof. The proof for global asymptotic stability of the endemic equilibrium point $Q^{*}$ follows directly from theorem (2). This is so, since for such an equilibrium point there exist a strict Lyapunov function $V(x)$ and hence by the Lyapunov's functional approach $Q^{*}$ is globally asymptotically stable.[1, 2] Also, since global asymptotic stability implies local asymptotic stability, we conclude that $Q^{*}$ is locally asymptotically stable. Hence the result follows.

\section{Conclusion}

A deterministic model $A$ that describes human population, where it is assumed that all state variables and parameters are non negative and that there is no disease induced death rate has been presented and its analysis has been dealt with using ideas of Lyapunov, Barbalart, Birkhoff and Rota. $[1,7,2]$ It has been shown that the system of equations that describes a model $A$ of $2 n$ finite compartments of susceptible and infected individuals, is bounded in a region $\Omega: \Omega=\left\{\left(S_{1}, I_{1}, S_{2}, I_{2}, S_{3}, I_{3}, \ldots, S_{n}, I_{n}\right): 0 \leq N(t) \leq k\right\}$.

In addition, it has been shown that if the endemic equilibrium point $Q^{*}$ exist then there exists a strict Lyapunov function $V(x)$ which has a global minimum at that equilibrium point $Q^{*}$. Moreover, by following the aforementioned ideas, it has been proved that $Q^{*}$ is both globally and locally asymptotically stable whenever it exist. 
Examples of models where we can apply the previously mentioned three theorems are that of modeling trichomoniasis infection and that of chancroid transmission dynamics. $[3,5,4,6]$ For these examples all state variables and parameters are assumed to be non-negative, since the models monitors human population. Moreover, the infections themselves do not kill, hence there is no disease induced death. Therefore the system of equations that describes or models trichomoniasis or chancroid transmission dynamics is dissipative, and for an endemic equilibrium point of such a system, there exist a strict Lyapunov function $V(x) \cdot[3,5,4,6]$ Using this, the conclusion that the endemic equilibrium point is globally and locally asymptotically stable for $R_{T}>1$ can be drawn for the "Transmission dynamics of trichomoniasis in bisexuals", for instance.[3]

\section{References}

[1] A.M. Lyapunov, Stability of Motions. Academic Press, New York and London (1966)

[2] A.M. Lyapunov, The General Problem of the Stability of motion. (A. T. Fuller trans.) Taylor and Francis, London (1992).

[3] P. Mafuta, J. Mushanyu, S. Mushayabasa, C.P. Bhunu, Transmission Dynamics of Trichomoniasis in bisexuals. World Journal of Modelling and Simulations 9(2013) 302-320.

[4] J. Mushanyu, P. Mafuta, S. Mushayabasa, C.P. Bhunu, Assessing the Impact of Educational Cam- paigns and Condom use in Chancroid Transmission Dynamics. World Journal of Modelling and Simulations (2013) submitted.

[5] C.P. Bhunu, S. Mushayabasa, Transmission Dynamics of Trichomonas vaginalis: A mathematical approach. Journal of Mathematical Analysis and Applications 379(2011) 852-860.

[6] C.P Bhunu, S. Mushayabasa, Chancroid Transmission Dynamics: A Mathematical Approach. The- ory in Bio-sciences 130(2011) 289-298.

[7] G. Birkhorff, G.C. Rota, Ordinary Differential Equations. Needham Heights, Ginn (1982). 